Draft VERSion May 29, 2018

Preprint typeset using $\mathrm{AT}_{\mathrm{E} X} \mathrm{X}$ style emulateapj v. 08/22/09

\title{
REDEFINING THE MISSING SATELLITES PROBLEM
}

\author{
Louis E. Strigari ${ }^{1}$, James S. Bullock ${ }^{1}$, Manoj Kaplinghat ${ }^{1}$, Juerg Diemand ${ }^{2,5}$, Michael Kuhlen ${ }^{3}$, Piero \\ $\mathrm{MADAU}^{2,4}$ \\ Draft version May 29, 2018
}

\begin{abstract}
Numerical simulations of Milky-Way size Cold Dark Matter (CDM) halos predict a steeply rising mass function of small dark matter subhalos and a substructure count that greatly outnumbers the observed satellites of the Milky Way. Several proposed explanations exist, but detailed comparison between theory and observation in terms of the maximum circular velocity $\left(V_{\max }\right)$ of the subhalos is hampered by the fact that $V_{\max }$ for satellite halos is poorly constrained. We present comprehensive mass models for the well-known Milky Way dwarf satellites, and derive likelihood functions to show that their masses within $0.6 \mathrm{kpc}\left(M_{0.6}\right)$ are strongly constrained by the present data. We show that the $M_{0.6}$ mass function of luminous satellite halos is flat between $\sim 10^{7}$ and $10^{8} M_{\odot}$. We use the "Via Lactea" N-body simulation to show that the $M_{0.6}$ mass function of CDM subhalos is steeply rising over this range. We rule out the hypothesis that the 11 well-known satellites of the Milky Way are hosted by the 11 most massive subhalos. We show that models where the brightest satellites correspond to the earliest forming subhalos or the most massive accreted objects both reproduce the observed mass function. A similar analysis with the newly-discovered dwarf satellites will further test these scenarios and provide powerful constraints on the CDM small-scale power spectrum and warm dark matter models.
\end{abstract}

Subject headings: Cosmology: dark matter, theory-galaxies

\section{INTRODUCTION}

It is now well-established that numerical simulations of Cold Dark Matter (CDM) halos predict many orders of magnitude more dark matter subhalos around Milky Way-sized galaxies than observed dwarf satellite galaxies (Klypin et al. 1999; Moore et al. 1999; Diemand et al. 2007). Within the context of the CDM paradigm, there are well-motivated astrophysical solutions to this 'Missing Satellites Problem' (MSP) that rely on the idea that galaxy formation is inefficient in the smallest dark matter halos (Bullock et al. 2000; Benson et al. 2002; Somerville 2002; Stoehr et al.|2002; Kravtsov et al. 2004; Moore et al. 2006; Gnedin \& Kravtsov 2006). However, from an observational perspective, it has not been possible to distinguish between these solutions.

A detailed understanding of the MSP is limited by our lack of a precise ability to characterize the dark matter halos of satellite galaxies. From an observational perspective, the primary constraints come from the velocity dispersion of $\sim 200$ stars in the population of dark matter dominated dwarf spheroidal galaxies (dSphs) (Wilkinson et al. 2004; Lokas et al. 2005; Munoz et al. 2005, 2006; Westfall et al. 2006; Walker et al.|2005, 2006; Sohn et al. 2006; Gilmore et al. 2007). However, the observed extent of the stellar populations in dSphs are $~$ $\mathrm{kpc}$, so these velocity dispersion measurements are only able to probe properties of the halos in this limited radial

\footnotetext{
${ }^{1}$ Center for Cosmology, Department of Physics \& Astronomy, University of California, Irvine, CA 92697

2 Department of Astronomy \& Astrophysics, University of California, Santa Cruz, CA 95064

${ }^{3}$ School of Natural Sciences, Institute for Advanced Study, Einstein Drive, Princeton, NJ 08540

${ }^{4}$ Max-Planck-Institut für Astrophysik, Karl-Schwarzschild-Str. 1, 85740 Garching, Germany.

${ }^{5}$ Hubble Fellow
}

regime.

From the theoretical perspective, dissipationless numerical simulations typically characterize subhalo counts as a function of the total bound mass or maximum circular velocity, $V_{\max }$. While robustly determined in simulations, global quantities like $V_{\max }$ are difficult to constrain observationally because dark halos can extend well beyond the stellar radius of a satellite. Indeed stellar kinematics alone provide only a lower limit on the halo $V_{\max }$ value (see below). This is a fundamental limitation of stellar kinematics that cannot be remedied by increasing the number of stars used in the velocity dispersion analysis (Strigari et al. 2007). Thus determining $V_{\max }$ values for satellite halos requires a theoretical extrapolation. Any extrapolation of this kind is sensitive to the predicted density structure of subhalos, which depends on cosmology, power-spectrum normalization, and the nature of dark matter (Zentner \& Bullock 2003).

Our inability to determinate $V_{\max }$ is the primary limitation to test solutions to the MSP. One particular solution states that the masses of the dSphs have been systematically underestimated, so that the $\sim 10$ brightest satellites reside systematically in the $\sim 10$ most massive subhalos (Stoehr et al. 2002; Havashi et al. 2003). A byproduct of this solution is that there must be a sharp mass cutoff at some current subhalo mass, below which galaxy formation is suppressed. Other solutions, based on reionization suppression, or a characteristic halo mass scale prior to subhalo accretion predict that the suppression comes in gradually with current subhalo mass (Bullock et al. 2000; Kravtsov et al. 2004; Moore et al. 2006).

In this paper, we provide a systematic investigation of the masses of the Milky Way satellites. We highlight that in all cases the total halo masses and maximum 
circular velocities are not well-determined by the data. We instead use the fact that the total cumulative mass within a characteristic radius $\sim 0.6 \mathrm{kpc}$ is much better determined by the present data (Strigari et al. 2007). We propose using this mass, which we define as $M_{0.6}$, as the favored means to compare to the dark halo population in numerical simulations. Unlike $V_{\max }, M_{0.6}$ is measured directly and requires no cosmology-dependent or dark-matter-model-dependent theoretical prior.

In the following sections, we determine the $M_{0.6}$ mass function for the Milky Way satellites, and compare it to the corresponding mass function measured directly in the high-resolution "Via Lactea" substructure simulation of Diemand et al. (2007). We rule out the possibility that there is a one-to- one correspondence between the 11 most luminous satellites and the most massive subhalos in Via Lactea. We find that MSP solutions based on reionization and/or characteristic halo mass scales prior to accretion are still viable.

\section{MILKY WAY SATELLITES}

Approximately 20 satellite galaxies can be classified as residing in MW subhalos. Of these, $\sim 9$ were discovered within the last two years and have very low luminosities and surface brightnesses (Willman et al. 2005; Willman et al. 2005; Belokurov et al. 2006, 2007; Zucker et al. 2006a,b). The lack of precision in these numbers reflects the ambiguity in the classification of the newly-discovered objects. The nine 'older' galaxies classified as dSphs are supported by their velocity dispersion, and exhibit no rotational motion (Mateo 1998). Two satellite galaxies, the Small Megallanic Cloud (SMC) and Large Megallanic Cloud (LMC), are most likely supported by some combination of rotation and dispersion. Stellar kinematics suggest that the LMC and SMC are likely the most massive satellite systems of the Milky Way.

We focus on determining the masses of the nine most well-studied dSphs. The dark matter masses of the dSphs are determined from the line-of-sight velocities of the stars, which trace the total gravitational potential. We assume a negligible contribution of the stars to the gravitational potential, which we find to be an excellent approximation. The $\mathrm{dSph}$ with the smallest mass-to-light ratio is Fornax, though even for this case we find that the stars generally do not affect the dynamics of the system (see Lokas 2002; Wu 2007, and below).

Under the assumptions of equilibrium and spherical symmetry, the radial component of the stellar velocity dispersion, $\sigma_{r}$, is linked to the total gravitational potential of the system via the Jeans equation,

$$
r \frac{d\left(\nu_{\star} \sigma_{r}^{2}\right)}{d r}=-\nu_{\star} V_{c}^{2}-2 \beta \nu_{\star} \sigma_{r}^{2} .
$$

Here, $\nu_{\star}$ is the stellar density profile, $V_{c}^{2}=G M(r) / r$ includes the total gravitating mass of the system, and the parameter $\beta(r)=1-\sigma_{\theta}^{2} / \sigma_{r}^{2}$ characterizes the difference between the radial $\left(\sigma_{r}\right)$ and tangential $\left(\sigma_{\theta}\right)$ velocity dispersions. The observable velocity dispersion is constructed by integrating the three- dimensional stellar radial velocity dispersion profile along the line-of-sight,

$$
\sigma_{\text {los }}^{2}(R)=\frac{2}{I_{\star}(R)} \int_{R}^{\infty}\left(1-\beta \frac{R^{2}}{r^{2}}\right) \frac{\nu_{\star} \sigma_{r}^{2} r d r}{\sqrt{r^{2}-R^{2}}},
$$

where $R$ is the projected radius on the sky. The surface density of stars in all dSphs are reasonably well-fit by a spherically-symmetric King profile (King 1962),

$$
I_{\star}(R) \propto\left[\left(1+\frac{R^{2}}{r_{k i n g}^{2}}\right)^{-1 / 2}-\left(1+\frac{r_{t}^{2}}{r_{k i n g}^{2}}\right)^{-1 / 2}\right]^{2},
$$

where $r_{t}$ and $r_{k i n g}$ are fitting parameters denoted as the tidal and core radii. ${ }^{6}$ The spherically symmetric stellar density can be obtained with an integral transformation of the surface density. From the form of Eq. (2), the normalization in Eq. (3) is irrelevant.

Some dSphs show evidence for multiple, dynamically distinct stellar populations, with each population described by its own surface density and velocity dispersion (Harbeck et al. 2001; Tolstoy et al. 2004; Westfall et al. 2006; McConnachie et al. 2006; Ibata et al. 2006). In a dSph with $i=1 \ldots N_{p}$ populations of stars, standard observational methods will sample a density-weighted average of the populations:

$$
\begin{aligned}
\nu_{\star} & =\sum_{i} \nu_{i} \\
\sigma_{r}^{2} & =\frac{1}{\nu_{\star}} \sum_{i} \nu_{i} \sigma_{r, \imath}^{2},
\end{aligned}
$$

where $\nu_{i}$ and $\sigma_{i}$ are the density profile and radial stellar velocity dispersion of stellar component $i$. In principle, each component has its own stellar velocity anisotropy profile, $\beta_{i}(r)$. In this case, Equation (1) is valid for $\nu_{\star}$ and $\sigma_{r}$ as long as an effective velocity anisotropy is defined as

$$
\beta(r)=\frac{1}{\nu_{\star} \sigma_{r}^{2}} \sum_{i} \beta_{i} \nu_{i} \sigma_{i}^{2}
$$

From these definitions, we also have $\sum_{\imath} I_{\star, \imath} \sigma_{l o s, \imath}^{2}=$ $I_{\star} \sigma_{\text {los }}^{2}$.

The conclusion we draw from this argument is that the presence of multiple populations will not affect the inferred mass structure of the system, provided that the velocity anisotropy is modeled as a free function of radius. Since the functional form of the stellar velocity anisotropy is unknown, we allow for a general, three parameter model of the velocity anisotropy profile,

$$
\beta(r)=\beta_{\infty} \frac{r^{2}}{r_{\beta}^{2}+r^{2}}+\beta_{0} .
$$

A profile of this form allows for the possibility for $\beta(r)$ to change from radial to tangential orbits within the halo, and a constant velocity anisotropy is recovered in the limit $\beta_{\infty} \rightarrow 0$, and $\beta_{0} \rightarrow$ const.

In Equations (11) and (2), the radial stellar velocity dispersion, $\sigma_{r}$, depends on the total mass distribution, and thus the parameters describing the dark matter density profile. Dissipation-less N-body simulations show that the density profiles of CDM halos can be characterized as

$$
\rho(\tilde{r})=\frac{\rho_{s}}{\tilde{r}^{\gamma}(1+\tilde{r})^{\delta}} ; \quad \tilde{r}=r / r_{s},
$$

6 Our results are insensitive to this particular parameterization of the light profile. 
where $r_{s}$ and $\rho_{s}$ set a radial scale and density normalization and $\gamma$ and $\delta$ parameterize the inner and outer slopes of the distribution. For dark matter halos unaffected by tidal interactions, the most recent high-resolution simulations find $\delta+\gamma \approx 3$ works well for the outer slope, while $0.7 \lesssim \gamma \lesssim 1.2$ works well down to $\sim 0.1 \%$ of halo virial radii (Navarro et al. 2004; Diemand et al. 2005). This interior slope is not altered as a subhalo loses mass from tidal interactions with the MW potential (Kazantzidis et al. 2004). The outer slope, $\delta$, depends more sensitively on the tidal interactions in the halo. The majority of the stripped material will be from the outer parts of halos, and thus $\delta$ of subhalo density profiles will become steeper than those of field halos. Subhalos are characterized by outer slopes in the range $2 \lesssim \delta \lesssim 3$.

Given the uncertainty in the $\beta(r)$ and $\rho(r)$ profiles, we are left with nine shape parameters that must be constrained via line-of-sight velocity dispersion measurements: $\rho_{s}, r_{s}, \beta_{0}, \beta_{\infty}, r_{\beta}, \gamma, \delta, r_{k i n g}$, and $r_{t}$. While the problem as posed may seem impossible, there are a number of physical parameters, which are degenerate between different profile shapes, that are well constrained. Specifically, the stellar populations constrain $V_{c}(r)$ within a radius comparable to the stellar radius $r_{t} \sim \mathrm{kpc}$. As a result, quantities like the local density and integrated mass within the stellar radius are determined with high precision (Strigari et al. 2006), while quantities that depend on the mass distribution at larger radii are virtually unconstrained by the data.

It is useful to determine the value of the radius where the constraints are maximized. The location of this characteristic radius is determined by the form of the integral in Eq. (2). We can gain some insight using the example of a power-law stellar distribution $\nu_{\star}(r)$, power-law dark matter density profile $\rho \propto r^{-\gamma_{\star}}$, and constant velocity anisotropy. The line-of-sight velocity dispersion depends on the three-dimensional stellar velocity dispersion, which can be written as

$$
\sigma_{r}^{2}(r)=\nu_{\star}^{-1} r^{-2 \beta} \int_{r}^{r_{t}} G \nu_{\star}(r) M(r) r^{2 \beta-2} \mathrm{~d} r \propto r^{2-\gamma_{\star}}
$$

From the shape of the King profile, the majority of stars reside at projected radii $r_{\text {king }} \lesssim R \lesssim r_{\mathrm{t}}$, where the stellar distribution is falling rapidly $\nu_{\star} \sim r^{-3.5}$. In this case, for $\beta=0$, the LOS component scales as $\sigma_{\text {los }}^{2}(R) \propto \int_{R}^{r_{t}} r^{-0.5-\gamma_{\star}}\left(r^{2}-R^{2}\right)^{-1 / 2} \mathrm{~d} r$ and is dominated by the mass and density profile at the smallest relevant radii, $r \sim r_{\text {king }}$. For $R \lesssim r_{\text {king }}, \nu_{\star} \propto r^{-1}$ and $\sigma_{\text {los }}^{2}$ is similarly dominated by $r \sim r_{\text {king }}$ contributions. We note that although the scaling arguments above hold for constant velocity anisotropies, they can be extended by considering anisotropies that vary significantly in radius. They are also independent of the precise form of $I_{\star}$, provided there is a scale similar to $r_{k i n g}$.

In Strigari et al. (2007), it was shown that typical velocity dispersion profiles best constrain the mass (and density) within a characteristic radius $\simeq 2 r_{k i n g}$. For example, the total mass within $2 r_{\text {king }}$ is constrained to within $\sim 20 \%$ for dSphs with $\sim 200$ line-of-sight velocities. Note that when deriving constraints using only the innermost stellar velocity dispersion and fixing the anisotropy to $\beta=0$, the characteristic radius for best constraints decreases to $\sim 0.5 r_{\text {king }}$ (e.g. Penarrubia et al.
2007).

As listed in Table 11. the Milky Way dSphs are observed to have variety of $r_{k i n g}$ values, but $r_{k i n g} \sim 0.3$ $\mathrm{kpc}$ is typical. The values of $r_{k i n g}$ and $r_{t}$ are taken from Mateo (1998). In order to facilitate comparison with simulated subhalos, we chose a single characteristic radius of $0.6 \mathrm{kpc}$ for all the dwarfs, and we represent the mass within this radius as $M_{0.6}=M(<0.6 \mathrm{kpc})$. The relative errors on the derived masses are unaffected for small variations in the characteristic radius in the range $\sim 1.5-2.5 r_{\text {king }}$. Deviations from a true King profile at large radius (near $r_{t}$ ) do not affect these arguments, as long as there is a characteristic scale similar to $r_{k i n g}$ in the surface density. The only dSph significantly affected by the choice of $0.6 \mathrm{kpc}$ as the characteristic radius is Leo II, which has $r_{t}=0.5 \mathrm{kpc}$. Since the characteristic radius is greater than twice $r_{k i n g}$, the constraints on its mass will be weakest of all galaxies (with the exception of Sagittarius, as discussed below).

\section{DARK MATTER HALO MASSES AT THE CHARACTERISTIC RADIUS}

We use the following data sets: Wilkinson et al. (2004); Munoz et al. (2005, 2006); Westfall et al. (2006); Walker et al. (2005, 2006); Sohn et al. (2006); Siegal et al. in preparation. These velocity dispersions are determined from the line-of-sight velocities of $\sim 200$ stars in each galaxy, although observations in the coming years will likely increase this number by a factor $\sim 5-10$. From the data, we calculate the $\chi^{2}$, defined in our case as

$$
\chi^{2}=\sum_{\imath=1}^{n} \frac{\left(\sigma_{o b s, \imath}-\sigma_{t h, \imath}\right)^{2}}{\epsilon_{\imath}^{2}} .
$$

Here $\sigma_{\text {obs }}^{2}$ is the observed velocity dispersion in each bin, $\sigma_{t h}^{2}$ is the theoretical value, obtained from Eq. (2), and $\epsilon_{\imath}^{2}$ are errors as determined from the observations.

It is easy to see that, when fitting to a single data set of $\sim 200$ stars, parameter degeneracies will be significant. However, from the discussion in section 2, $M_{0.6}$ is well-determined by the LOS data. To determine how well $M_{0.6}$ is constrained, we construct likelihood functions for each galaxy. When thought of as a function of the theoretical parameters, the likelihood function, $\mathcal{L}$, is defined as the probability that a data set is acquired given a set of theoretical parameters. In our case $\mathcal{L}$ is a function of the parameters $\gamma, \delta, r_{s}, \rho_{s}$, and $\beta_{0}, \beta_{\infty}, r_{\beta}$, and is defined as $\mathcal{L}=e^{-\chi^{2} / 2}$. In writing this likelihood function, we assume that the errors on the measured velocity dispersions are Gaussian, which we find to be an excellent approximation to the errors for a dSph system (Strigari et al. 2007). We marginalize over the parameters $\gamma, \delta, r_{s}, \rho_{s}$, and $\beta_{0}, \beta_{\infty}, r_{\beta}$ at fixed $M_{0.6}$, and the optimal values for $M_{0.6}$ are determined by the maximum of $\mathcal{L}$.

We determine $\mathcal{L}$ for all nine $\mathrm{dSphs}$ with velocity dispersion measurements. For all galaxies we use the full published velocity dispersion profiles. The only galaxy that does not have a published velocity dispersion profile is Sagittarius, and for this galaxy we use the central velocity dispersion from Mateo (1998). The mass modeling of Sagittarius is further complicated by the fact that it is experiencing tidal interactions with the MW (Ibata et al. 


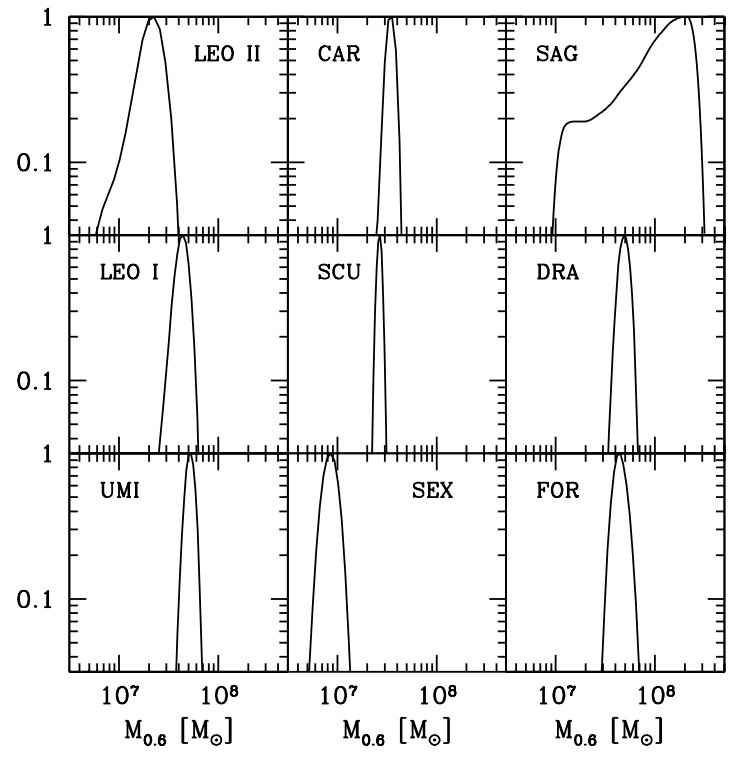

FIG. 1.- The likelihood functions for the mass within $0.6 \mathrm{kpc}$ for the nine dSphs, normalized to unity at the peak.

1997; Ma jewski et al. 2003), so a mass estimate from the Jeans equation is not necessarily reliable. We caution that in this case the mass we determine is likely only an approximation to the total mass of the system.

We determine the likelihoods by marginalizing over the following ranges of the velocity anisotropy, inner and outer slopes: $-10<\beta_{0}<1,-10<\beta_{\infty}<1$, $0.1<r_{\beta}<10 \mathrm{kpc}, 0.7<\gamma<1.2$, and $2<\delta<3$. As discussed above, these ranges for the asymptotic inner and outer slopes are appropriate because we are considering CDM halos. It is important to emphasize that these ranges are theoretically motivated and that observations alone do not demand such restrictive choices. It is possible to fit all of the dSphs at present with a constant density cores with scale-lengths $\sim 100 \mathrm{pc}$ (Strigari et al. 2006; Gilmore et al. 2007), although the data by no means demand such a situation. Though we consider inner and outer slopes in the ranges quoted above, our results are not strongly affected if we widen these intervals. For example, we find that if we allow the inner slope to vary down to $\gamma=0$, the widths of the likelihoods are only changed by $\sim 10 \%$. This reflects the fact that there is a negligible degeneracy between $M_{0.6}$ and the inner and outer slopes.

We are left to determine the regions of $\rho_{s}$ and $r_{s}$ parameter space to marginalize over. In all $\mathrm{dSphs}$, there is a degeneracy in this parameter space, telling us that it is not possible to derive an upper limit on this pair of parameters from the data alone (Strigari et al. 2006). While this degeneracy is not important when determining constraints on $M_{0.6}$, it is the primary obstacle in determining $V_{\max }$. From the fits we present below, we find that the lowest $r_{s}$ value that provides an acceptable fit is $\sim 0.1 \mathrm{kpc}$, and we use this as the lower limit in all cases. In our fiducial mass models, we conservatively restrict the maximum value of $r_{s}$ using the known distance to each dSph. In this case, we use $0.1 \mathrm{kpc}<r_{s}<D / 2$, where $D$ is the distance to the dSph.

In Figure 1 we show the $M_{0.6}$ likelihood functions for all of the dSphs. As is shown, we obtain strong con-

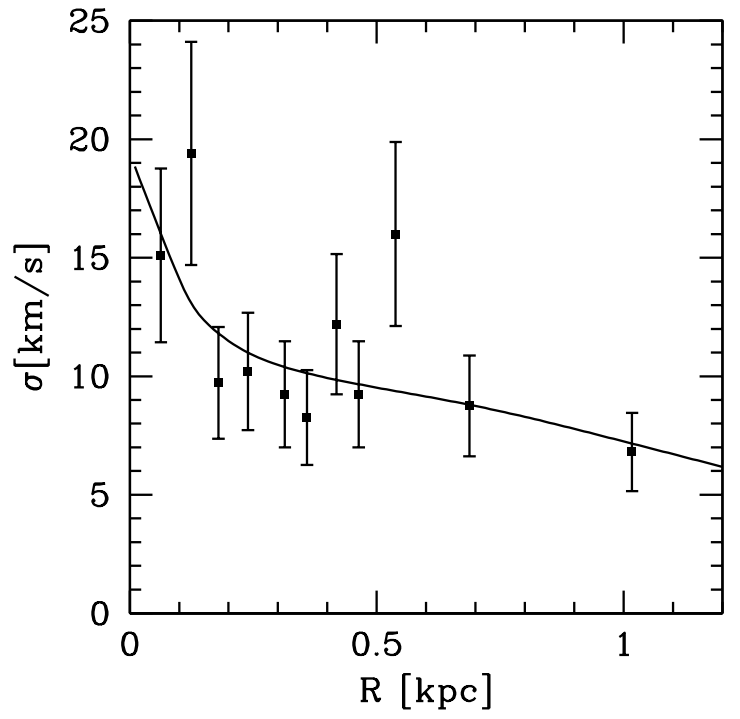

FIG. 2.- The velocity dispersion for Ursa Minor as a function of radial distance, along with the model that maximizes the likelihood function.

straints on $M_{0.6}$ in all cases except Sagittarius, for which we use only a central velocity dispersion. Table 1 summarizes the best fitting $M_{0.6}$ values for each dwarf. The quoted errors correspond to the points where the likelihood falls to $10 \%$ of its peak value. The upper panel of Figure 3 shows $M_{0.6}$ values for each dwarf as a function of luminosity. In Figure 2 we show an example of the velocity dispersion data as a function of radial distance for Ursa Minor, along with the model that maximizes the likelihood function. For all galaxies, we find $\chi^{2}$ per degree of freedom values $\lesssim 1$.

The maximum likelihood method also allows us to constrain the mass at other radii spanned by the stellar distribution. The sixth column of Table 1 provides the integrated mass within each dwarf's King tidal radius. This radius roughly corresponds to the largest radius where a reasonable mass constraint is possible. As expected, the mass within $r_{t}$ is not as well determined as the mass within $2 r_{k i n g}$. From these masses we are able to determine the mass-to-light ratios within $r_{t}$, which we present in the seventh column of Table 1. In the bottom panel of Figure 3, we show mass-to-light ratios within $r_{t}$ as a function of dwarf luminosity. We see the standard result that the observable mass-to-light ratio increases with decreasing luminosity (Mateo 1998). Note, however, that our results are inconsistent with the idea that all of the dwarfs have the same integrated mass within their stellar extent. We note that for Sagittarius, we can only obtain a lower limit on the total mass-to-light ratio.

The last two columns in Table 1 list constraints on $V_{\max }$ for the dSphs. Column 8 shows results for an analysis with limits on $r_{s}$ as described above. In this case, the integrated mass within the stellar radius is constrained by the velocity dispersion data, but the halo rotation velocity curve, $V_{c}(r)$, can continue to rise as $r$ increases beyond the stellar radius in an unconstrained manner. The result is that the velocity dispersion data alone provide only a lower limit on $V_{\max }$.

Stronger constraints on $V_{\max }$ can be obtained if we limit the range of $r_{s}$ by imposing a cosmology-dependent prior on the dark matter mass profile. CDM simula- 
TABLE 1

Parameters Describing Milky Way Satellites.

\begin{tabular}{l|lllllllc}
\hline \hline \multicolumn{1}{c|}{ Galaxy } & $\begin{array}{l}r_{\text {king }} \\
{[\mathrm{kpc}]}\end{array}$ & $\begin{array}{c}r_{t} \\
{[\mathrm{kpc}]}\end{array}$ & $\begin{array}{c}\mathrm{L}_{V} \\
{\left[10^{6} \mathrm{~L} \odot\right]}\end{array}$ & $\begin{array}{l}\text { Mass }<0.6 \mathrm{kpc} \\
{\left[10^{7} \mathrm{M} \odot\right]}\end{array}$ & $\begin{array}{l}\text { Mass }<r_{t} \\
{\left[10^{7} \mathrm{M} \odot\right]}\end{array}$ & $\begin{array}{l}M\left(<r_{t}\right) / L \\
{\left[\mathrm{M}_{\odot} / L_{\odot}\right]}\end{array}$ & $\begin{array}{l}V_{\max }\left[\mathrm{km} \mathrm{s}^{-1}\right] \\
\text { (w/o prior) }\end{array}$ & $\begin{array}{c}V_{\max }\left[\mathrm{km} \mathrm{s}^{-1}\right] \\
\text { (with theory prior) }\end{array}$ \\
\hline Draco & 0.18 & 0.93 & 0.26 & $4.9_{-1.3}^{+1.4}$ & $14_{-4.2}^{+7.0}$ & 530 & $>22$ & $28_{-9}^{+21}$ \\
Ursa Minor & 0.30 & 1.50 & 0.29 & $5.3_{-1.3}^{+1.3}$ & $23_{-11}^{+16}$ & 790 & $>21$ & $26_{-6}^{+12}$ \\
Leo I & 0.20 & 0.80 & 4.79 & $4.3_{-1.6}^{+1.6}$ & $8.5_{-2.8}^{+4.5}$ & 106 & $>14$ & $19_{-5}^{+13}$ \\
Fornax & 0.39 & 2.70 & 15.5 & $4.3_{-1.1}^{+2.7}$ & $44_{-29}^{+3 .}$ & 28 & $>20$ & $25_{-5}^{+5}$ \\
Leo II & 0.19 & 0.52 & 0.58 & $2.1_{-1.1}^{+1.6}$ & $2.1_{-1.1}^{+1.6}$ & 128 & $>17$ & $9_{-1}^{+3}$ \\
Carina & 0.26 & 0.85 & 0.43 & $3.4_{-1.0}^{+0.7}$ & $6.7_{-2.5}^{+2.3}$ & 82 & $>13$ & $15_{-3}^{+5}$ \\
Sculptor & 0.28 & 1.63 & 2.15 & $2.7_{-0.4}^{+0.4}$ & $15_{-1.5}^{+0.7}$ & 68 & $>20$ & $14_{-2}^{+2}$ \\
Sextans & 0.40 & 4.01 & 0.50 & $0.9_{-0.3}^{+0.4}$ & $13_{-5.8}^{+1.1}$ & 260 & $>8$ & $9_{-1}^{+1}$ \\
Sagittarius & 0.3 & 4.0 & 18.1 & $20_{-20}^{+10}$ & $>20$ & $>11$ & $>19$ & -
\end{tabular}

Note. - Determination of the mass within $0.6 \mathrm{kpc}$ and the maximum circular velocity for the dark matter halos of the dSphs. The errors are determined as the location where the likelihood function falls off by $90 \%$ from its peak value. For Sagittarius, no reliable estimate of $V_{\max }$ with the CDM prior could be determined. The CDM prior is determined using the concordance cosmology with $\sigma_{8}=0.74, n=0.95$ (see text for details).

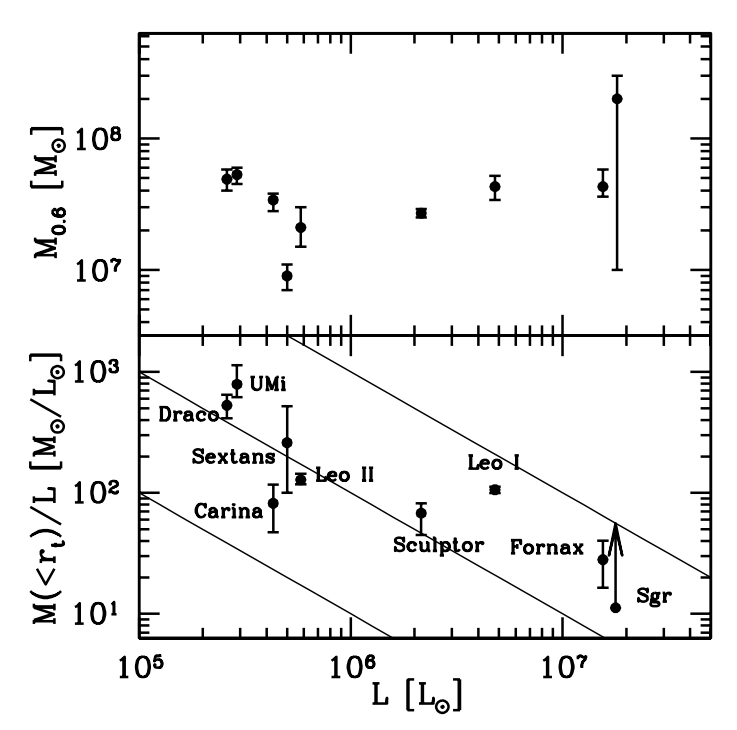

FIG. 3.- The mass within $0.6 \mathrm{kpc}$ (upper) and the mass-to-light ratios within the King tidal radius (lower) for the Milky Way dSphs as a function of dwarf luminosity. The error-bars here are defined as the locations where the likelihoods fall to $40 \%$ of the peak values (corresponding to $\sim 1 \sigma$ errors). The lines denote, from top to bottom, constant values of mass of $10^{7}, 10^{8}, 10^{9} M_{\odot}$.

tions have shown that there is a correlation between $V_{\max }$ and $r_{\max }$ for halos, where $r_{\max }$ is the radius where the circular velocity peaks. Because subhalo densities will depend on the collapse time and orbital evolution of each system, the precise $V_{\max }-r_{\max }$ relation is sensitive to cosmology (e.g. $\sigma_{8}$ ) and the formation history of the host halo itself (e.g. Zentner \& Bullock 2003; Power 2003; Kazantzidis et al. 2004; Bullock \& Johnston 2005; Bullock \& Johnston 2006). When converted to the relevant halo parameters, the imposed $V_{\max } r_{\max }$ relation can be seen as a theoretical prior on CDM halos, restricting the parameter space we need to integrate over. In order to illustrate the technique, we adopt $\log _{10}\left(r_{\max }\right)=1.35\left(\log _{10}\left(V_{\max } / \mathrm{km} \mathrm{s}^{-1}\right)-1\right)-0.196 \mathrm{kpc}$ with a scatter of 0.2 in $\log _{10}$, as measured from simulated subhalos within the Via Lactea host halo (Diemand et al. 2007). This simulation is for a LCDM cosmology with $\sigma_{8}=0.74$ and $n=0.95$. The scatter in the subhalo mass function increases at the very high mass end, which reflects the fact that these most massive subhalos are those that are accreted most recently (Zentner \& Bullock 2003; van den Bosch et al. 2005). However, as we show below our results are not strongly dependent on the large scatter at the high mass end.

Column 9 in Table 1 shows the allowed subhalo $V_{\max }$ values for the assumed prior. Note that in most cases, this prior degrades the quality of the fit, and the likelihood functions peak at a lower overall value. The magnitude of this effect is not large except for the cases of Leo II and Sagittarius. For Leo II, the peak likelihood with the prior occurs at a value that is below the $10 \%$ likelihood for the case without a prior on $r_{s}$ (i.e. the data seem to prefer a puffier subhalo than would be expected in CDM). For Sagittarius, we are unable to obtain a reasonable fit within a subhalo that is typical of those expected. This is not too surprising. Sagittarius is being tidally disrupted and its dark matter halo is likely atypical.

We emphasize that the $V_{\max }$ determinations listed in Column 9 are driven by theoretical assumptions, and can only be fairly compared to predictions for this specific cosmology ( $\mathrm{LCDM}, \sigma_{8}=0.74$ ). The $M_{0.6}$ values in Column 5 are applicable for any theoretical model, including non-CDM models, or CDM models with any normalization or power spectrum shape.

\section{COMPARISON TO NUMERICAL SIMULATIONS}

The recently-completed Via Lactea run is the highestresolution simulation of galactic substructure to date, containing an order of magnitude more particles than its predecessors (Diemand et al. 2007). As mentioned above, Via Lactea assumes a LCDM cosmology with $\sigma_{8}=0.74$ and $n=0.95$. For a detailed description of the simulation, see Diemand et al. (2007). For our purposes, the most important aspect of Via Lactea is its ability to resolve the mass of subhalos on length scales of the characteristic radius $0.6 \mathrm{kpc}$. In Via Lactea, the force resolution is $90 \mathrm{pc}$ and the smallest well-resolved length scale is $300 \mathrm{pc}$, so that the mass within $0.6 \mathrm{kpc}$ is well-resolved in nearly all subhalos. Due to the choice of time steps we expect the simulation to underestimate local densities in the densest regions (by about $10 \%$ at 


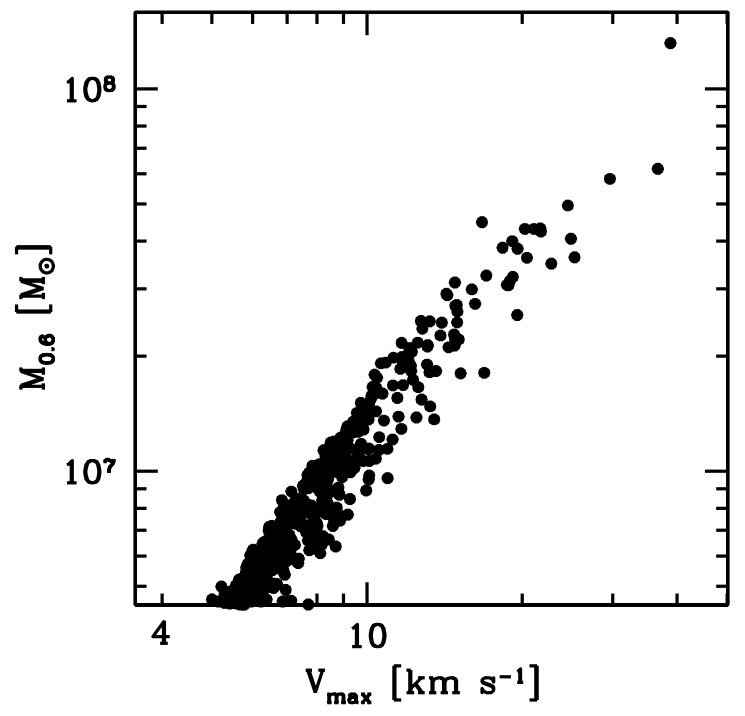

FIG. 4. - The mass within $0.6 \mathrm{kpc}$ versus the maximum circular velocity for the mass ranges of Via Lactea subhalos corresponding to the population of satellites we study.

densities of $\left.9 \times 10^{7} \mathrm{M}_{\odot} / \mathrm{kpc}^{3}\right)$. There is only one subhalo with a higher local density than this at $0.6 \mathrm{kpc}$. For this subhalo, $\rho(r=0.6 \mathrm{kpc})=1.4 \times 10^{8} \mathrm{M}_{\odot} / \mathrm{kpc}^{3}$, so its local density might be underestimated by up to $10 \%$, and the errors in the enclosed mass might be $\sim 20 \%$ (Diemand et al. 2005). For all other subhalos the densities at $0.6 \mathrm{kpc}$ are well below the affected densities, and the enclosed mass should not be affected by more than $10 \%$ by the finite numerical resolution.

We define subhalos in Via Lactea to be the self-bound halos that lie within the radius $R_{200}=389 \mathrm{kpc}$, where $R_{200}$ is defined to enclose an average density 200 times the mean matter density. We note that in comparing to the observed MW dwarf population, we could have conservatively chosen subhalos that are restricted to lie within the same radius as the most distant MW dSph $(250 \mathrm{kpc})$. We find that this choice has a negligible effect on our conclusions - it reduces the count of small halos by $\sim 10 \%$.

In Figure 4, we show how $M_{0.6}$ relates to the more familiar quantity $V_{\max }$ in Via Lactea subhalos. We note that the relationship between subhalo $M_{0.6}$ and $V_{\max }$ will be sensitive to the power spectrum shape and normalization, as well as the nature of dark matter (Bullock et al. 2001; Zentner \& Bullock 2003). The relationship shown is only valid for the Via Lactea cosmology, but serves as a useful reference for this comparison.

Given likelihood functions for the $\mathrm{dSph} M_{0.6}$ values, we are now in position to determine the $M_{0.6}$ mass function for Milky Way (MW) satellites and compare this to the corresponding mass function in Via Lactea. For both the observations and the simulation, we count the number of systems in four mass bins from $4 \times 10^{6}<M_{0.6}<$ $4 \times 10^{8} \mathrm{M}_{\odot}$. This mass range is chosen to span the $M_{0.6}$ values allowed by the likelihood functions for the MW satellites. We assume that the two non-dSph satellites, the LMC and SMC, belong in the highest mass bin, corresponding to $M_{0.6}>10^{8} \mathrm{M}_{\odot}$ (Harris \& Zaritskv 2006; van der Marel et al. 2002).

In Figure 5 we show resulting mass functions for MW satellites (solid) and for Via Lactea subhalos (dashed,

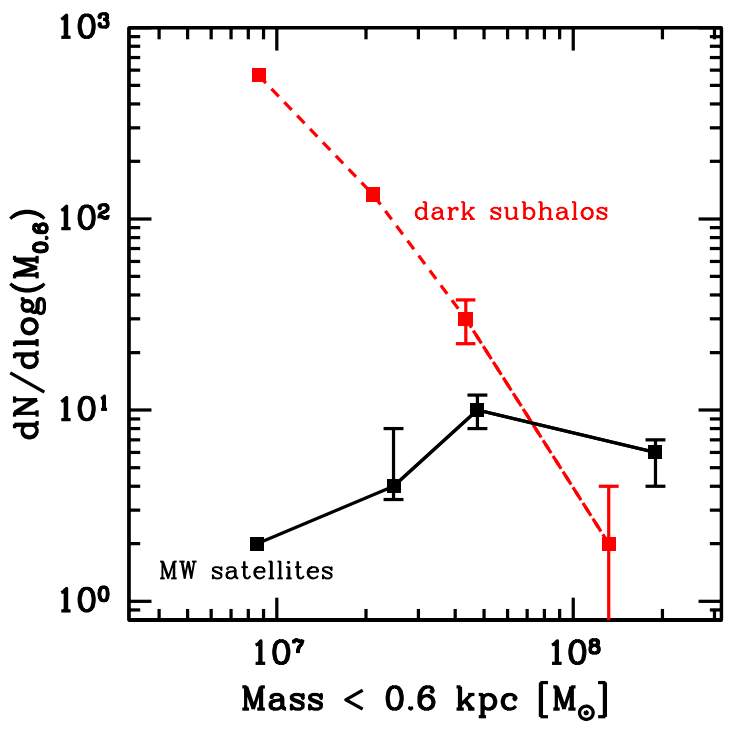

FIG. 5. - The $M_{0.6}$ mass function of Milky Way satellites and dark subhalos in the Via Lactea simulation. The red (short-dashed) curve is the total subhalo mass function from the simulation. The black (solid) curve is the median of the observed satellite mass function. The error-bars on the observed mass function represent the upper and lower limits on the number of configurations that occur with a probability of $>10^{-3}$.

with Poisson error-bars). For the MW satellites, the central values correspond to the median number of galaxies per bin, which are obtained from the maximum values of the respective likelihood functions. The error-bars on the satellite points are set by the upper and lower configurations that occur with a probability of $>10^{-3}$ after drawing 1000 realizations from the respective likelihood functions. As seen in Figure 5, the predicted dark subhalo mass function rises as $\sim M_{0.6}^{-2}$ while the visible MW satellite mass function is relatively flat. The lowest mass bin $\left(M_{0.6} \sim 9 \times 10^{6} M_{\odot}\right)$ always contains 1 visible galaxy (Sextans). The second-to-lowest mass bin $\left(M_{0.6} \sim 2.5 \times 10^{7} M_{\odot}\right)$ contains between 2 and 4 satellites (Carina, Sculptor, and Leo II). The fact that these two lowest bins are not consistent with zero galaxies has important implications for the Stoehr et al. (2002) solution to the MSP: specifically, it implies that the 11 well-known MW satellites do not reside in subhalos that resemble the 11 most massive subhalos in Via Lactea.

To further emphasize this point, we see from Figure 5 that the mass of the 11th most massive subhalo in Via Lactea is $4 \times 10^{7} \mathrm{M}_{\odot}$. From the likelihood functions in Figure 1. Sextans, Carina, Leo II, and Sculptor must have values of $M_{0.6}$ less than $4 \times 10^{7} \mathrm{M}_{\odot}$ at $99 \%$ c.l., implying a negligible probability that all of these dSphs reside in halos with $M_{0.6}>4 \times 10^{7} \mathrm{M}_{\odot}$.

Using the $M_{0.6}$ mass function of MW satellites, we can test other CDM-based solutions to the MSP. Two models of interest are based on reionization suppression (Bullock et al. 2000; Moore et al. 2006) and on there being a characteristic halo mass scale prior to subhalo accretion (Diemand et al. 2007). To roughly represent these models, we focus on two subsamples of Via Lactea subhalos: the earliest forming (EF) halos, and the largest mass halos before they were accreted (LBA) into the host halo. As described in Diemand et al. (2007), the LBA sample is defined to be the 10 subhalos that had 


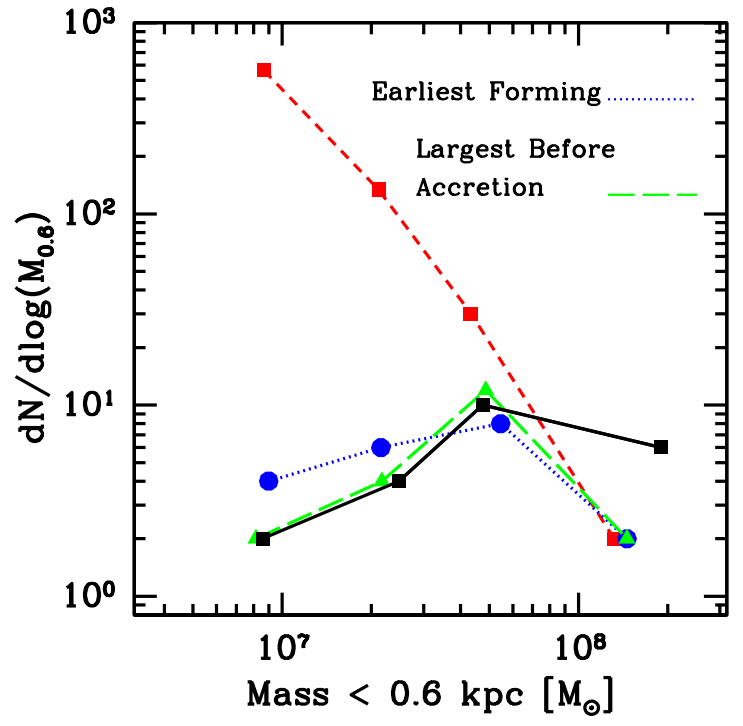

FIG. 6. - The solid and dashed curves show the MW satellites and dark subhalos in Via Lactea, respectively. These lines are reproduced from Figure 5 with error-bars suppressed for clarity. The blue (dotted) curve represents the ten earliest forming halos in Via Lactea, and the green (long-dashed) curve represents the 10 most massive halos before accretion into the Milky Way halo.

the highest $V_{\max }$ value throughout their entire history. These systems all had $V_{\max }>37.3 \mathrm{kms}^{-1}$ at some point in their history. The EF sample consists of the 10 subhalos with $V_{\max }>16.2 \mathrm{~km} \mathrm{~s}^{-1}$ (the limit of atomic cooling) at $z=9.6$. The Kravtsov et al. (2004) model would correspond to a selection intermediate between EF and LBA. In Figure 6 we show the observed mass function of MW satellites (solid, squares) along with the EF (dotted, triangles) and LBA (long-dashed, circles) samples. We conclude that both of these models are in agreement with the MW satellite mass function. Future observations and quantification of the masses of the newly-discovered MW satellites will enable us to do precision tests of the viable MSP solutions. Additionally, once the capability to do numerical simulations of substructure in warm dark matter models becomes a reality, the $M_{0.6}$ mass function will provide an invaluable tool to place constraints on WDM models.

\section{SUMMARY AND DISCUSSION}

We have provided comprehensive dark matter mass constraints for the 9 well-studied dSph satellite galaxies of the Milky Way and investigated CDM-based solutions for the missing satellite problem in light of these constraints. While subhalo $V_{\max }$ values are the traditional means by which theoretical predictions quantify substructure counts, this is not the most direct way to confront the observational constraints. Specifically, $V_{\text {max }}$ is poorly constrained by stellar velocity dispersion measurements, and can only be estimated by adopting cosmology-dependent, theoretical extrapolations. We argue the comparison between theory and observation is best made using the integrated mass within a fixed physical radius comparable to the stellar extent of the known satellites, $\sim 0.6 \mathrm{kpc}$. This approach is motivated by Strigari et al. (2007) who showed that the mass within two stellar King radii is best constrained by typical velocity dispersion data.
Using $M_{0.6}$ to represent the dark matter mass within a radius of $0.6 \mathrm{kpc}$, we computed $M_{0.6}$ likelihood functions for the MW dSphs based on published velocity dispersion data. Our models allow for a wide range of underlying dark matter halo profile shapes and stellar velocity dispersion profiles. With this broad allowance, we showed that the $M_{0.6}$ for most dwarf satellites is constrained to within $\sim 30 \%$.

We derived the $M_{0.6}$ mass function of MW satellites (with error bars) and compared it to the same mass function computed directly from the Via Lactea substructure simulation. While the observed $M_{0.6}$ mass function of luminous satellites is relatively flat, the comparable CDM subhalo mass function rises as $\sim M_{0.6}^{-2}$. We rule out the hypothesis that all of the well-known Milky Way satellites strictly inhabit the most massive CDM subhalos. If luminosity does track current subhalo mass, this would only be possible if the subhalo population of the Milky Way were drastically different than that predicted in CDM. However, we show that other plausible CDM solutions are consistent with the observed mass function. Specifically, the earliest forming subhalos have a flat $M_{0.6}$ mass function that is consistent with the satellite subhalo mass function. This would be expected if the population of bright dwarf spheroidals corresponds to the residual halo population that accreted a significant mount of gas before the epoch of reionization (Bullock et al. 2000). We also tested the hypothesis that the present dwarf spheroidal population corresponds to the subhalos that were the most massive before they fell into the MW halo (Kravtsov et al. 2004). This hypothesis is also consistent with the current data.

In deriving the $M_{0.6}$ mass function for this paper we have set aside the issue of the most- recently discovered population of MW dwarfs. We aim to return to this issue in later work, but it is worth speculating on the expected impact that these systems would have on our conclusions. If we had included the new systems, making $\sim 20$ satellites in all, would it be possible to place these systems in the $\sim 20$ most massive subhalos in Via Lactea? Given the probable mass ranges for the new dwarfs, we find that this is unlikely. We can get a rough estimate of their masses from their observed luminosities. We start by considering the mass-to-light ratios of the known dSph population from figure 3 and from Mateo (1998). If we assume that the other dwarfs have similar $M / L$ range, we can assign a mass range for each of them. In all cases, the new MW dwarfs are approximately 1 to 2 orders of magnitude smaller in luminosity than the well-known dSph population. Using the central points for the known dSphs, we obtain $M_{0.6} / L$ spanning the range from $3-230$. Considering the width of the likelihoods, we can allow a slightly larger range, $2-350$. If we place the new dwarfs in this latter range, the uncertainty in their masses is $(2-350) L M_{\odot} / L_{\odot}$. Even with this generous range we expect most of the new dwarfs have $M_{0.6} \lesssim 10^{7} \mathrm{M}_{\odot} \cdot{ }^{7}$

The discovery of more members of the MW group,

7 These estimates are in rough agreement with recent determinations from stellar velocity dispersion measurements in the new dwarfs, as presented by N. Martin and J. Simon at the 3rd Irvine Cosmology Workshop, March 22-24, 2007, http://www.physics.uci.edu/Astrophysical-Probes/ 
and the precise determination of the $M_{0.6}$ mass function, could bring the status of the remaining viable MSP solutions into sharper focus. These measurements would also provide important constraints on warm dark matter models or on the small scale power spectrum in CDM.

\section{ACKNOWLEDGMENTS}

We thank Jason Harris, Tobias Kaufmann, Savvas Koushiappas, Andrey Kravtsov, Steve Majewski, Nicolas Martin, Josh Simon, and Andrew Zentner for discussions on this topic. We thank Mike Siegal for sharing his Leo II data. LES is supported in part by a Gary McCue postdoctoral fellowship through the Center for Cosmology at the University of California, Irvine. L.E.S., J.S.B., and M.K. are supported in part by NSF grant AST-0607746. M.K. acknowledges support from PHY-0555689. J. D. acknowledges support from NASA through Hubble Fellowship grant HST-HF-01194.01 awarded by the Space Telescope Science Institute, which is operated by the Association of Universities for Research in Astronomy, Inc., for NASA, under contract NAS 5-26555. P.M. acknowledges support from NASA grants NAG5-11513 and NNG04GK85G, and from the Alexander von Humboldt Foundation. The Via Lactea simulation was performed on NASA's Project Columbia supercomputer system.
Belokurov, V. et al. 2006, Astrophys. J., 647, L111

- 2007, Astrophys. J., 654, 897

Benson, A. J., Lacey, C. G., Baugh, C. M., Cole, S., \& Frenk, C. S. 2002, Mon. Not. Roy. Astron. Soc., 333, 156

Bullock, J. S. \& Johnston, K. V. 2005, ApJ, 635, 931

Bullock, J. S. \& Johnston, K. V. 2006, To appear in the proceedings of 'Island Universes: Structure and Evolution of Disk Galaxies', ed. R. de Jong (SPringer: Dordrecht)

Bullock, J. S., Kravtsov, A. V., \& Weinberg, D. H. 2000, Astrophys. J., 539, 517

Bullock, J. S. et al. 2001, Mon. Not. Roy. Astron. Soc., 321, 559

Diemand, J., Kuhlen, M., \& Madau, P. 2007, Astrophys. J., 657, 262

Diemand, J., Kuhlen, M., \& Madau, P. 2007, astro-ph/0703337

Diemand, J., Zemp, M., Moore, B., Stadel, J., \& Carollo, M. 2005, Mon. Not. Roy. Astron. Soc., 364, 665

Gilmore, G. et al. 2007, astro-ph/0703308

Gnedin, N. Y. \& Kravtsov, A. V. 2006, Astrophys. J., 645, 1054

Harbeck, D. et al. 2001, Astron. J., 122, 3092

Harris, J. \& Zaritsky, D. 2006, AJ, 131, 2514

Hayashi, E., Navarro, et al. 2003, ApJ, 584, 541

Ibata, R., Chapman, S., Irwin, M., Lewis, G., \& Martin, N. 2006, Mon. Not. Roy. Astron. Soc. Lett., 373, L70

Ibata, R. A., Wyse, R. F. G., Gilmore, G., Irwin, M. U., \& Suntzeff, N. B. 1997, Astron. J., 113, 634

Kazantzidis, S., Mayer, L., Mastropietro, C., Diemand, J., Stadel, J., \& Moore, B. 2004, ApJ, 608, 663

King, I. 1962, Astron. J., 67, 471

Klypin, A., Kravtsov, A. V., Valenzuela, O., \& Prada, F. 1999, ApJ, 522, 82

Kravtsov, A. V., Gnedin, O. Y., \& Klypin, A. A. 2004, Astrophys. J., 609, 482

Lokas, E. L. 2002, Mon. Not. Roy. Astron. Soc., 333, 697

Lokas, E. L., Mamon, G. A., \& Prada, F. 2005, Mon. Not. Roy. Astron. Soc., 363, 918

Majewski, S. R., Skrutskie, M. F., Weinberg, M. D., \& Ostheimer, J. C. 2003, Astrophys. J., 599, 1082

Mateo, M. 1998, Ann. Rev. Astron. Astrophys., 36, 435

McConnachie, A. W., Penarrubia, J., \& Navarro, J. F. 2006, astro-ph/0608687

\section{REFERENCES}

Moore, B., Diemand, J., Madau, P., Zemp, M., \& Stadel, J. 2006, Mon. Not. Roy. Astron. Soc., 368, 563

Moore, B. et al. 1999, ApJ, 524, L19

Munoz, R. R. et al. 2005, Astrophys. J., 631, L137

—. 2006, Astrophys. J., 649, 201

Navarro, J. F. et al. 2004, Mon. Not. Roy. Astron. Soc., 349, 1039

Penarrubia, J., McConnachie, A., \& Navarro, J. F. 2007, astro-ph/0701780

Power, C. 2003, Ph.D. Thesis, University of Durham

Sohn, S. T. et al. 2006, astro-ph/0608151

Somerville, R. S. 2002, ApJ, 572, L23

Stoehr, F., White, S. D. M., Tormen, G., \& Springel, V. 2002,

Mon. Not. Roy. Astron. Soc., 335, L84

Strigari, L. E., Bullock, J. S., \& Kaplinghat, M. 2007, Astrophys. J., 657, L1

Strigari, L. E. et al. 2006, Astrophys. J., 652, 306

Strigari, L. E. et al. 2006, astro-ph/0611925

Tolstoy, E. et al. 2004, Astrophys. J., 617, L119

van den Bosch, F. C., Tormen, G., \& Giocoli, C. 2005, Mon. Not. Roy. Astron. Soc., 359, 1029

van der Marel, R. P., Alves, D. R., Hardy, E., \& Suntzeff, N. B. 2002, AJ, 124, 2639

Walker, M. G., Mateo, M., Olszewski, E. W., Bernstein, R. A., Wang, X., \& Woodroofe, M. 2005, AJ in press (astro-ph/0511465)

Walker, M. G., Mateo, M., Olszewski, E. W., Pal, J. K., Sen, B., \& Woodroofe, M. 2006, ApJ, 642, L41

Westfall, K. B., Majewski, S. R., Ostheimer, J. C., Frinchaboy, P. M., Kunkel, W. E., Patterson, R. J., \& Link, R. 2006, AJ, 131,375

Wilkinson, M. I. et al. 2004, Astrophys. J., 611, L21

Willman, B. et al. 2005, AJ, 129, 2692

Willman, B. et al. 2005, Astrophys. J., 626, L85

Wu, X. 2007, astro-ph/0702233

Zentner, A. R. \& Bullock, J. S. 2003, Astrophys. J., 598, 49

Zucker, D. B. et al. 2006a, Astrophys. J., 650, L41

—. 2006b, Astrophys. J., 643, L103 